\title{
Hepatic Cystic Echinococcosis in Australia: An Update on Diagnosis and Management
}

\author{
Ben Keong FRACS ${ }^{1}$, Bruce Wilkie MA MBBS ${ }^{2}$, Tom Sutherland MMed FRANZCR ${ }^{3}$, \\ Adrian Fox FRACS ${ }^{2}$ \\ ${ }^{1}$ Department of Upper Gastrointestinal Surgery, St George Hospital, Kogarah NSW, \\ Australia \\ ${ }^{2}$ Department of Surgery, Eastern Health, Melbourne, Victoria, Australia \\ ${ }^{3}$ Medical Imaging Department, St Vincent's Hospital, Melbourne, Victoria, Australia
}

Keywords: hydatid; hepatic; surgery; ultrasound; zoonosis

Word Count (inc. headings):

Abstract: 225

Text: 2,851

References: 861

Total: 3,937

\section{Correspondence:}

Dr Bruce Wilkie, Eastern Health, 8 Arnold Street, Box Hill, Victoria 3128, Australia

Email: brucedwilkie@gmail.com Tel: +613 88049999

Disclosure: There are no conflicts of interest. This paper is not based on communication to a society or meeting.

This is the author manuscript accepted for publication and has undergone full peer review but has not been through the copyediting, typesetting, pagination and proofreading process, which may lead to differences between this version and the Version of Record. Please cite this article as doi: $10.1111 /$ ans.14117

This article is protected by copyright. All rights reserved. 


\section{Abstract}

Background: Echinococcosis is an uncommonly encountered zoonotic disease caused by the taeniid Echinococcus. The only endemic species in Australia, E granulosus, forms cysts in the liver in seventy percent of cases.

Aim: To review the literature to provide an evidence-based narrative update on the diagnosis and management of hepatic cystic echinococcosis in Australia.

Methods: We reviewed the literature, utilising multiple research databases and citation tracking. Original research and review articles examining the diagnosis and management of hydatid disease in adults, published prior to 2016, and in the English language were included in our review.

Results: Ultrasound is the gold-standard screening test, whilst computed tomography has a role in emergency presentations and screening for multi-organ involvement. Magnetic resonance imaging is preferred second-line imaging and better demonstrates biliary involvement. Medical therapy or PAIR may be appropriate in selected cases however, surgery remains the definitive treatment for active, large $(>5 \mathrm{~cm})$, symptomatic or complicated cysts. A variety of surgical techniques have been described, including conservative, radical and minimally-invasive procedures. There is currently no consensus approach; surgical modality should be tailored to patient factors, relevant anatomy, local facilities and surgeons' expertise.

This article is protected by copyright. All rights reserved. 
Conclusion: Diagnosis and therapy in hepatic hydatid cysts (HHC) have been significantly advanced by imaging, interventional radiology and surgical approaches in recent years. Surgery remains the mainstay of treatment for large, active, complicated or symptomatic hepatic hydatid cysts.

\section{Background}

Echinococcosis is a zoonotic, cyst-forming disease caused by the larval stage of the taeniid Echinococcus. Humans are inadvertent intermediate hosts in the life cycle of the tapeworm, which matures in the small bowel of canids (dogs and dingoes) and reproduces in the solid viscera of grazing animals (sheep, cattle, and macropodids). Humans become infected via ingesting eggs shed in dog faeces.

E granulosus is the only species endemic to Australia and human infection is termed cystic echinoccocosis (CE). CE may produce a spectrum of disease from a singular, asymptomatic cyst to severe, complicated infection. Complicated disease typically results from cyst rupture, cyst contents communicating with the biliary tree or peritoneal cavity, secondary infection, or the mass effect exerted on adjacent organs. Cysts grow $1-5 \mathrm{~cm}$ per year and consist of a fibrous host-derived outer layer (pericyst), an acellular, laminated membrane (ectocyst) and a nucleated inner layer (endocyst) ${ }^{1}$.

This article is protected by copyright. All rights reserved. 
Alveolar echinococcosis (AE), caused by E multilocularis, is extremely rare in Australia. AE may be acquired in remote areas of Europe, Asia, China, Canada, and Alaska ${ }^{2}$. After a latent period of 10-15 years, untreated AE develops a progressive course with significant mortality $(>90 \%)$. Both CE and AE are considered re-emerging zoonoses of public health importance ${ }^{3}$. This review refers only to the diagnosis and management of hepatic CE.

\section{$\underline{\text { Epidemiology in Australia }}$}

E granulosus was introduced into Australia with sheep-grazing ${ }^{4}$. The G1 genotype ('sheep strain') established viable reservoirs in native wallabies and kangaroos and has been readily disseminated by dingoes and wild dogs ${ }^{5}$. 80-100 cases of echinococcosis are diagnosed across Australia each year ( 0.4 per 100,000 population), though the true prevalence of CE is unknown due to the asymptomatic nature of many infections and frequent underreporting. Rates in rural areas are higher than equivalent urban populations ( 0.9 per 100,000), and rural Aboriginal people are disproportionally represented $(5.5 \text { per } 100,000)^{6}$. The highest rates recorded on mainland Australia have been in rural north-east and south-east NSW (23.5 per $100,000)^{6}$

Risk factors for CE include previous episodes of echinococcosis, occupational and domestic exposure to dogs, and travel to or from endemic areas. Case-control studies suggest the risk is highest in settlements of $<500$ people, and increases with the number of dogs kept and number of years of coexistence with them ${ }^{7}$. The fatality rate of $\mathrm{CE}$ is dependent on the site of 
infection and availability of appropriate medical treatment. The overall mortality rate is 0.2 per 100,000 population $^{8}$.

Simple control measures (i.e. dry dog foods, three-monthly praziquantel dog-deworming, hand hygiene, legislation and education) have significantly reduced the transmission of Echinococcus between domestic dogs and humans. Novel control measures in development include a new dog-stage vaccine and EG95 recombinant sheep vaccine ${ }^{4}$. Tasmania was declared hydatid-free in 1996 thanks to a government-sponsored eradication campaign ${ }^{9}$. A similar program in New Zealand resulted in no new cases being reported in persons under 19 years of age since $1977^{2}$.

\section{$\underline{\text { Clinical Features }}$}

Population-based screening studies suggest that half of CE infections are asymptomatic ${ }^{8}$. Signs or symptoms relate to the site and size of the cyst within organs, rate of growth and mass effect, hosts' immunologic response or susceptibility of cysts to secondary infection ${ }^{10}$. Due to the portal venous migration of parasites, $70 \%$ of $\mathrm{CE}$ occurs primarily in the liver. $\mathrm{CE}$ typically presents with a solitary cyst in a single organ (usually liver or lungs), however between $20-40 \%$ of cases have either multiple cysts or multi-organ involvement ${ }^{11}$.

Common clinical features of HHC include a hepatic mass, right hypochondrial pain, nausea and vomiting. Chronic infection may present with biliary colic, hepatomegaly, fistulae, abscesses, jaundice, ascites, portal hypertension, and IVC or Budd-Chiari syndromes. 
Complicated HHC are almost always symptomatic, and broadly categorised into either rupture or secondary infection ${ }^{12}$. Perforated cysts communicating with the biliary tree may present with cholangitis, while acute intraperitoneal rupture may result in anaphylaxis.

\section{Imaging}

Radiological investigations are able to provide definitive diagnosis of $\mathrm{CE}$ in the majority of cases and modalities may include ultrasound (US), computed tomography (CT) and magnetic resonance imaging (MRI).

US is considered the gold-standard screening test. US appearance of hepatic CE has been described using a standardised World Health Organisation (WHO) classification (Table 1) ${ }^{13}$. CE 1-3 have pathognomonic features, exhibiting either 'hydatid sand' (suspended protoscoleces), 'honeycombing' (daughter cysts), or the 'water-lily sign' (floating internal membranes). Pericystic calcification may be present in inactive cysts and can be seen as a hyperechoic 'arch-shaped' contour with acoustic shadowing. While CE 1-3 do not generally require further diagnostic imaging, screening of abdominopelvic organs with US and a chest $\mathrm{x}$-ray is advised.

CT may be useful screening for extrahepatic disease, identifying small cysts $(\mathrm{e} 1 \mathrm{~cm}$ in diameter), and observing the internal architecture of cysts, liver parenchyma and biliary tree. CT has a high specificity and sensitivity (approaching 96\%) when used in conjunction with serology $^{8}$, and may identify intraperitoneal rupture or the cyst-wall thickening redolent of 
secondary infection. Both US and CT can detect cyst wall discontinuity indicative of cyst rupture, however small communications are best described via direct cholangiography ${ }^{12}$.

MRI is superior to $\mathrm{CT}$ for demonstrating the internal composition of cysts and potential biliary involvement. Diffusion-weighted MRI in particular is useful in differentiating HHC from simple cysts $^{14}$. A retrospective study in patients who had CT/MRI in addition to US, showed that the level of agreement with US for CE 1-4 was higher for MRI than for $\mathrm{CT}^{15}$. CT had a higher level of agreement for CE 5, where cystic calcification was more accurately measured. With superior soft tissue resolution, MRI reproduces US features of HHC more accurately than CT and is preferable as second-line imaging.

\section{$\underline{\text { Laboratory Investigations }}$}

Nonspecific leukopenia, thrombocytopenia and derangement of liver function may be present. Neutrophilia is indicative of secondary infection and eosinophilia suggests cyst rupture. Serology is useful in the primary work-up or where imaging is atypical, however false-negative results are common (between 20-40\%) due to low or undetectable circulating antibodies in uncomplicated disease ${ }^{8}$. Primary antibody tests may also produce false positives with autoimmune disease, cirrhosis, and malignancy ${ }^{16}$.

Commonly utilised primary antibody tests are the indirect haemagglutination antibody test (IHAT) and latex agglutination test (LAT). IHAT and LAT have quoted sensitivities of $80 \%$ in liver-only disease. Enzyme-linked immunosorbent assay for IgG antibodies (IgG-ELISA)

This article is protected by copyright. All rights reserved. 
is considered the gold-standard with a sensitivity of up to $93 \%$ for liver-only disease ${ }^{8}$. Secondary antibody testing either via Arc 5, IgG4-ELISA or immunoblotting may be used in the absence of diagnostic imaging. Secondary antibody tests are less sensitive than primary tests but highly specific. The Arc 5 test has a sensitivity of $50-60 \%$ but is highly specific $(97.2 \%)$ for taeniid infections ${ }^{17}$.

\section{Fine-needle Aspiration}

US-guided, transhepatic fine-needle aspiration (FNA) of suspected HHC is generally considered safe under appropriate albendazole cover (at least four days prior and one month following cyst puncture). It is contraindicated in large superficially located cysts under high internal pressure, patients with high total $\operatorname{IgE}$ titres, or with a history of severe allergic reactions and anaphylaxis ${ }^{8}$.

\section{Chemotherapy}

Systemic benzimidazole anti-helminthic drugs (albendazole and mebendazole) have proven safe and effective in the treatment echinococcosis, with randomised trials demonstrating the superiority of albendazole when compared to mebendazole ${ }^{18}$. A continuous three-month course of albendazole $(10-15 \mathrm{mg} / \mathrm{kg}$ in two divided doses) may be appropriate as primary therapy for small $(<5 \mathrm{~cm})$, uncomplicated CE 1 and CE 3a HHC. Multiple small cysts, deep parenchymal or peritoneal cysts may also be amenable to drug therapy alone ${ }^{19}$. Analyses of large datasets $(n=3760)$ have suggested that albendazole monotherapy may result in a 
complete response in $30 \%$ of cases, with another $40-50 \%$ showing objective evidence of cyst degradation at follow-up ${ }^{20}$.

A randomised-controlled trial of 55 patients determined that a 3-month preoperative course of albendazole resulted in $68 \%$ of cysts showing echographic evidence of total cyst membrane disintegration, which correlated with non-viability $(94 \%, \mathrm{p}=0.015)$ of cysts at time of surgery when compared to controls ${ }^{21}$. Other studies have suggested however that a 6-month course of albendazole results in a cure in only $18 \%$ of cases and recommended as monotherapy only in non-surgical candidates ${ }^{22}$. All patients undergoing extended benzimidazole therapy should have routine second-weekly monitoring for bone-marrow suppression and impairment of liver function ${ }^{8}$.

Albendazole should be used as an adjunct to surgery to reduce recurrence. Therapy should commence at least 1 month (ideally 3 months) prior to surgery and 3 months postoperatively. A randomised study of 72 patients compared surgery alone with 12 weeks of pre-operative albendazole ${ }^{23}$. At the time of surgery, viable protoscolices were found in all cysts in the surgery-only group compared with only $5.5 \%$ in the albendazole group. There was no recurrence in the albendazole group compared to $16.6 \%$ in the surgery-only group with five-year follow-up. A further randomised study $(n=64)$ demonstrated recurrence rates of $18.75 \%$ and $4.16 \%$ in surgery-only vs albendazole groups respectively ${ }^{24}$. The mean period of post-operative recurrence in one study of 172 patients was $23.3 \pm 5.3$ months (range, 8$48)^{25}$

This article is protected by copyright. All rights reserved. 


\section{$\underline{\text { PAIR }}$}

PAIR (percutaneous aspiration, irrigation with scolicide and re-aspiration) has been utilised safely and effectively in selected cases. A study of 336 patients over a 23 -years found that of the 48 patients treated with PAIR, $36(66.6 \%)$ experienced complete resolution of the disease $^{26}$. Another series reported 26 patients with 32 HHC successfully treated with PAIR and followed-up with 6-monthly serology and annual imaging for 10 years $^{27}$.

The main complication associated with PAIR or intraoperative scolicidal instillation is chemical sclerosing cholangitis and biliary stricturing where cysts communicate with the biliary tree. Other potential risks associated with PAIR include bleeding, infection, intraperitoneal spillage of cyst contents, and biliary fistulae. Allergic reactions and anaphylaxis $(<1 \%)$ may also occur ${ }^{28}$.

\section{$\underline{\text { Scolicides }}$}

Scolicides may be used intraoperatively or for PAIR. In vitro studies have demonstrated Betadine $\AA$ (povidone-iodine or PVP-I), 20\% hypertonic saline, 10\% Savlon ${ }^{\circledR}(1.5 \% / 0.15 \%$ cetrimide-chlorhexidine), and $95 \%$ ethyl alcohol to be effective scolicides $^{29}$. Cetrimidechlorhexidine is scolicidal at all concentrations from $0.15 / 0.015 \%$ to $1.5 \% / 0.15 \%$ at both five and ten minutes post-instillation. A $50 \%$ dilution of Betadine ${ }^{\circledR}$ and $20 \%$ hypertonic saline is also effective within five minutes of instillation. Historically, formalin was a highly efficacious scolicide, but carries an untenable risk of chemical cholangitis and should never 
be used. Alcohol can cause caustic irritation to bile duct epithelium and systemic toxicity. PVP-I carries some risk of PVP storage disease, renal injury, sterile peritonitis and sclerosing $\operatorname{serositis}^{29}$.

\section{Surgery}

Surgery is the principal treatment for large, active, symptomatic or complicated hepatic CE. It is indicated for all symptomatic cysts, CE2-CE3b cysts, cysts $>5 \mathrm{~cm}$, cysts with multiple daughter vesicles, superficially located or infected cysts, cysts communicating with the biliary tree or cysts exerting mass effect on adjacent organs. There are several viable surgical approaches: anatomical hepatectomy, pericystectomy, and open cystectomy with or without omentoplasty. Every effort should be made to protect the peritoneal cavity by avoiding intraoperative spillage of cyst contents and the use of scolicide-soaked surgical packs where appropriate $^{16}$.

\section{Conservative vs Radical Surgery}

In a retrospective review over a 25 -year period in a single hepatobiliary unit, 214 patients underwent conservative surgery where all or part of the pericyst remained (external drainage, marsupialisation, or omentoplasty) while 240 patients had radical surgery (hepatic resection or cystopericystectomy) $)^{30}$. Patient demographics and overall mortality were similar howver operative morbidity, overall hospital stay and recurrence rate was substantially higher in the conservative surgery group when compared to radical surgery $(79.9 \%$ vs $16.2 \%, p<0.001$, 33.7 vs 13.8 days, $\mathrm{p}<0.001$ and $30.4 \%$ vs $1.2 \%, \mathrm{p}=0.016$ respectively). 
The increased surgical morbidity was attributed to the residual pericyst lining which resulted in bile leaks, fistulae, and abscess formation. In addition, reoperation in these situations was more complex. Thus, the authors' preferred procedure was cystopericystectomy, with complete removal of the cyst and pericyst without opening the cavity.

Another retrospective study compared patients undergoing radical surgery (pericystectomy or left lobe segmentectomy) with conservative surgery (partial cystectomy and external drainage plus or minus omentoplasty) $)^{31}$. All patients underwent pre- and post-operative albendazole therapy. With radical cystectomy, the cyst was removed with $1 \mathrm{~cm}$ of liver parenchyma without opening the cavity. The conservative technique involved removal of as much of the anterior cyst as possible and evacuation of cystic contents. There were no differences in patient demographics, the number, size and location of cysts, nor rates of post-operative complication.

Recurrence was lower in the radical surgery group ( $0 \%$ vs $17 \%, \mathrm{p}=0.045)$. The authors recommended however a more conservative technique in the elderly, patients with higher ASA scores, and deeply located $(\mathrm{e} 10 \mathrm{~cm})$, bilobar or posterior cysts. Favourable characteristics for radical surgery included younger patients, cysts located anterosuperiorly or in the left lateral segment, exophytic cysts, cysts e $5 \mathrm{~cm}$ diameter, and the procedure being performed by an experienced hepatobiliary surgeon. 


\section{$\underline{\text { Laparoscopic vs Open }}$}

The largest published series comparing laparoscopic to open surgery for HHC included 333 patients over a 10 -year period at a single-centre ${ }^{32}$. In this study, 59 patients underwent a laparoscopic approach and 172 patients underwent open surgery. Indications for laparoscopic surgery included lesions located in liver segments other than segment I or VII, corticalisation of the liver surface, and absence of cyst rupture. Procedures performed laparoscopically were either Lagrot partial pericystectomy (54 cases) or total pericystectomy (5 cases).

Indications for the conventional open procedure included intrabiliary or intraperitoneal rupture and cysts located in segment VII of the liver. The procedures performed were Lagrot partial pericystectomy (which involves resection of the externalised extrahepatic pericyst "roof" to the border with the liver parenchyma, leaving the cavity/remaining intrahepatic pericyst in-situ) and drainage in the majority, with total pericystectomy, left lobectomy, or left hemihepatectomy in the remainder. The two groups were similar in terms of overall mortality cyst location, size and type. Complications in both arms of the study included collections in the residual cavity and biliary fistulae, while the conventional surgery group also had wound-related complications. Hospital stay was longer in the conventional group (11.7 vs 6.42 days, $\mathrm{p}<0.001)$. No recurrences were observed in either group. Confounding factors included higher rate of obesity $(24.4 \%$ vs $10.7 \%, \mathrm{p}=0.032)$ in the conventional group, as well as more radical procedures when compared to the laparoscopic group (20.9 vs $8.4 \%$, $\mathrm{p}=0.041)$ 
A systematic review of twenty-two retrospective studies examined 666 patients who had laparoscopic surgery for $\mathrm{HHC}^{33}$. Laparoscopic surgery was always conservative, being mainly unroofing of the cyst with partial pericystectomy. There was an average complication rate of $13.1 \%$, conversion to open in $4 \%$ of cases, average hospital stay of 4.6 days and overall recurrence rate of $1.6 \%$. In the absence of randomised-controlled trials, this study suggests that laparoscopy is a safe and feasible.

\section{Complicated Disease}

Complicated disease most commonly results from cyst rupture or secondary infection. All ruptured cysts are predisposed to secondary infection and should be managed as cholangitis. Trauma is the most common aetiology of perforation ${ }^{34}$. Cyst diameter over $9.5 \mathrm{~cm}$ correlates with intraperitoneal or intrabiliary perforation. A case control study $(n=372)$ identified that younger patients $(36.32 \pm 11.86$ years $)$ with large, superficially located cysts are predisposed to intraperitoneal rupture whilst older patients $(50.26 \pm 13.88$ years $)$ with large, multiple or bilobar cysts are at increased risk of spontaneous intrabiliary perforation ${ }^{34}$.

Post-operatively, bile leaks are more common in large cysts, with $79 \%$ of cysts e $7.5 \mathrm{~cm}$ in diameter likely to have cysto-biliary fistulae ${ }^{16}$. Communications can be identified intraoperatively by using dye or cholangiography. Most communications can be managed with suture; sphincterotomy alone is considered inadequate. Bile leaks should be managed conservatively wherever possible, though reoperation, biliary-intestinal anastomosis or liver resection may ultimately be required ${ }^{35}$. 


\section{$\underline{\text { Conclusion }}$}

Hydatid disease in Australia remains a relevant surgical and public health problem due to the potential serious complications and rural population exposed to infection. Simple control measures have been highly effective at reducing the incidence of the disease and a range of targeted control measures can be employed in hyperendemic areas to reduce the risk of human infection. Given that hydatid disease will be present on mainland Australia for the foreseeable future, it is imperative that general and hepatobiliary surgeons are aware of the various diagnostic and therapeutic approaches to best manage the disease.

Ultrasound has proven safety, utility and reliability in the diagnosis and follow-up of hepatic CE. CT is most useful in emergency presentations, and may also be useful for identifying small cysts and screening for multi-organ involvement. MRI is generally better at demonstrating the cystic components of $\mathrm{HHC}$ and potential biliary involvement. FNA and PAIR may be utilised safely in selected cases.

Serology is useful in the primary work-up of CE. It is recommended that seronegative and seropositive patients with non-diagnostic US proceed to further imaging, repeat serology and consideration of FNA to exclude differential diagnoses such as tuberculosis, abscesses and neoplasms. Seronegative patients with suggestive imaging should proceed to treatment if symptomatic. Post-operative or post-chemotherapy surveillance with US is recommended at 3-6 monthly intervals for up to 3 years. 
In all confirmed cases, an extended, continuous course of albendazole $(10-15 \mathrm{mg} / \mathrm{kg}$ orally, in divided doses, twice daily) for 3 months pre-operatively and 3 months post-operatively is recommended. Albendazole alone may be appropriate for selected cases and should always be used as an adjunct to surgery or PAIR unless contraindicated. Once biliary communications have been excluded, safe and effective scolicidal agents for intraoperative use include $20 \%$ hypertonic saline, Betadine ${ }^{\circledR}$ and Savlon ${ }^{\circledR}$, while $95 \%$ ethyl alcohol or hypertonic saline may be used for PAIR ${ }^{30}$.

Surgery remains the only definitive treatment for large, active, symptomatic or complicated HHC. A variety of surgical techniques have been described and there is no currently consensus approach. Surgery should to be tailored to patient factors, relevant anatomy, local facilities and surgeons' expertise. Radical surgery does have advantages over more conservative methods with reduced post-operative complications and rates of recurrence, while a laparoscopic approach has been shown to be both safe and technically feasible.

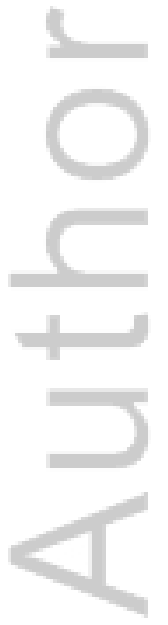




\section{Bibliography}

1. Chau GY, Lee RC, Lui YW, Wu CW. Hepatobiliary and pancreatic: Hepatic hydatid disease. $J$. Gastroenterol. Hepatol. 2004; 19(11): 1320.

2. Moro PS, Peter M. Echinococcosis: a review. Int. J. Infect. Dis., 2009. 13(2): 125-133.

3. Jenkins DJ, Romig T, Thompson RCA. Emergence/re-emergence of Echinococcus spp.—a global update. Int. J. Parasitol. 2005; 35(11-12): 1205-1219.

4. Jenkins DJ. Hydatid control in Australia: where it began, what we have achieved and where to from here. Int. J. Parasitol. 2005; 35(7): 733-740.

5. Barnes TS, Morton JM, Coleman GT. Clustering of hydatid infection in macropodids. Int. J. Parasitol. 2007; 37(8-9): 943-952.

6. Jenkins DJ, Waikagul J, Cross JH. Cystic echinococcosis in Australia: The current situation. Southeast Asian J. Trop. Med. Public Health. 2004; 35: 183-188.

7. Eckert J, Deplazes P. Biological, Epidemiological, and Clinical Aspects of Echinococcosis, a Zoonosis of Increasing Concern. Clin. Microbiol. Rev. 2004; 17(1): 107-135.

8. Pawłowski ZS, Eckert J, Vuitton DA et al. Echinococcosis in humans: clinical aspects, diagnosis and treatment. In: WHO/OIE Manual on Echinococcosis in Humans and Animals: A Public Health Problem of Global Concern, Eckert J (Ed) 2001: 20-72.

9. O'Hern, JA, Cooley L, A description of human hydatid disease in Tasmania in the post-eradication era. Med. J. Aust. 2013; 199(2): 117.

10. McManus DP, Zhang W, Li J, Bartley PB. Echinococcosis. Lancet, 2003; 362(9392): 1295-1304. 11. Moro P, Schantz APM, Echinococcosis: a review. Int. J. Infect. Dis. 2009; 13(2): 125-133. 12. Marti-Bonmati L, Serrano F, Complications of hepatic hydatid cysts: Ultrasound, computed tomography, and magnetic resonance diagnosis. Gastrointest. Radiol. 1990; 15(1): 119-125. 
13. WHO Informal Working Group. International classification of ultrasound images in cystic echinococcosis for application in clinical and field epidemiological settings. Acta Tropica 2003; 85(2): 253-261.

14. Inan N, Arslan A, Akansel G et al. Diffusion-weighted imaging in the differential diagnosis of simple and hydatid cysts of the liver. Am. J. Roentgenol., 2007. 189(5): 1031-1036.

15. Stojkovic M, Rosenberger K, Kauczor HU, Junghanss T, Hosch W. Diagnosing and staging of cystic echinococcosis: how do CT and MRI perform in comparison to ultrasound. PLoS Negl. Trop. Dis. 2012; 6(10): e1880.

16. Brunetti E, Kern P, Vuitton D.A. Expert consensus for the diagnosis and treatment of cystic and alveolar echinococcosis in humans. Acta Tropica 2010; 114(1): 1-16.

17. Zhang W, Li J, McManus DP. Concepts in immunology and diagnosis of hydatid disease. Clin. Microbiol. Rev. 2003; 16(1): 18-36.

18. Franchi, C., B.D. Vico, and A. Teggi, Long-Term Evaluation of Patients with Hydatidosis Treated with Benzimidazole Carbamates. Clinical Infectious Diseases, 1999. 29(2): 304-309.

19. Moro PL. Treatment of echinococcosis. In: UpToDate, Post TW (Ed), Waltham, MA, 2015.

20. Horton RJ. Albendazole in treatment of human cystic echinococcosis: 12 years of experience. Acta Tropica 1997; 64(1): 79-93.

21. Gil-Grande LA, Rodriguez-Caabeiro F, Prieto JG, et al. Randomised controlled trial of efficacy of albendazole in intra-abdominal hydatid disease. Lancet 1993; 342(8882): 1269-1272.

22. Kapan S, Turhan AN, Kalayci MU, Alis H, Aygun E. Albendazole is not effective for primary treatment of hepatic hydatid cysts. J. Gastrointest. Surg. 2008; 12(5): 867-871.

23. Shams UB, Arif SH, Malik AA, Khaja AR, Dass TA, Naikoo ZA. Role of albendazole in the management of hydatid cyst liver. Saudi J. Gastroenterol. 2011; 17(5): 343-347. 
24. Arif SH, Shams UB, Wani NA, et al. Albendazole as an adjuvant to the standard surgical management of hydatid cyst liver. Int. J. Surg. 2008; 6(6): 448-451.

25. Kapan M, Kapan S, Goksoy E, Perek S, Kol E. Postoperative Recurrence in Hepatic Hydatid Disease. J. Gastrointest. Surg. 2006; 10(5): 734-739.

26. El Sheikh A, Al-Malki A, El Sheikh MA, Al Robayan A. Non-surgical management in 336 patients of hydatid disease: 23 years experience at Riyadh Military Hospital.

Hepatogastroenterol. 2011; 58(106): 336-346.

27. Yasawy MI, Mohammed AE, Bassam S, Karawi MA, Shariq S. Percutaneous aspiration and drainage with adjuvant medical therapy for treatment of hepatic hydatid cysts. World. J.

Gastroenterol. 2011; 17(5): 646-650.

28. Golemanov B, Grigorov N, Mitova R, et al. Efficacy and Safety of PAIR for Cystic Echinococcosis: Experience on a Large Series of Patients from Bulgaria. Am. J. Trop. Med. Hyg. 2011; 84(1): 48-51.

29. Besim H, Karayalcin K, Hamamci O, Gungor C, Korkmaz A. Scolicidal agents in hydatid cyst surgery. HPB Surg. 1998; 10(6): 347-351.

30. Tagliacozzo S, Miccini M, Amore Bonapasta S, Gregori M, Tocchi A, Surgical treatment of hydatid disease of the liver: 25 years of experience. Am. J. Surg. 2011; 201(6): 797-804.

31. Akbulut S, Senol A, Ekin A, Bakir S, Bayan K, Dursun M. Radical vs conservative surgery for hydatid liver cysts: experience from single centre. World J. Gastroenterol. 2010; 16(8): 953-959.

32. Zaharie F, Bartos D, Mocan L, Zaharie R, Iancu C, Tomus C. Open or laparoscopic treatment for hydatid disease of the liver? A 10-year single-institution experience. Surg. Endosc 2013; 27(6): 21102116.

33. Citgez B, Battal M, Cipe G, Karatepe O, Muslumanoglu M. Feasibility and safety of laparoscopic hydatid surgery: a systematic review. Hepatogastroenterol 2013; 60(124): 784-788. 
34. Akcan A, Sozuer E, Akyildiz H, Ozturk A, Atalay A, Yilmaz Z. Predisposing factors and surgical outcome of complicated liver hydatid cysts. World J Gastroenterol 2010; 16(24): 3040-3048.

35. Aydin U, Yazici P, Onen Z, et al. The optimal treatment of hydatid cyst of the liver: radical surgery with a significant reduced risk of recurrence, Turk. J. Gastroenterol. 2008; 19: 33-39

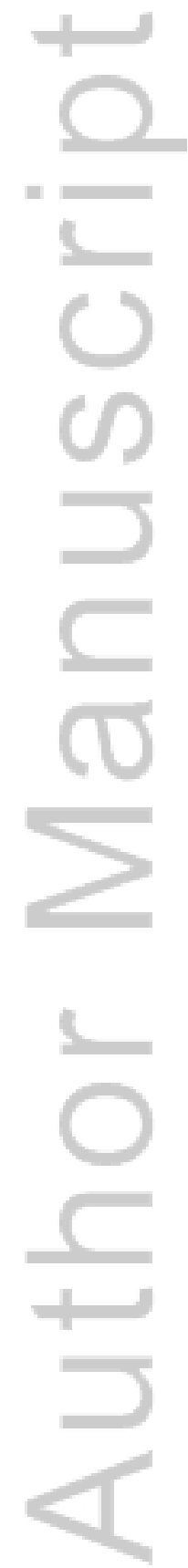




\begin{tabular}{|l|l|}
\hline Table 1. WHO CE classification on ultrasound \\
\hline Classification & Ultrasound features \\
\hline CL $^{\dagger}$ & $\begin{array}{l}\text { Unilocular cystic lesion with uniform anechoic content } \\
\text { Wall may not be visible }\end{array}$ \\
\hline CE 1 & $\begin{array}{l}\text { Unilocular, simple cyst with anechoic content } \\
\text { Cyst wall visible } \\
\text { 'Hydatid sand' or 'snowflake sign' present }\end{array}$ \\
\hline CE 2 & $\begin{array}{l}\text { Multi-vesicular, multi-septate cysts } \\
\text { Daughter cysts indicated by 'honeycomb-like' structures where present } \\
\text { Cysts wall normally visible }\end{array}$ \\
\hline CE 3 & $\begin{array}{l}\text { Unilocular cysts that may contain daughter cysts } \\
\text { Anechoic content with detachment of laminated membrane from cysts } \\
\text { wall } \\
\text { Floating membranes may exhibit 'water-lily sign' (CE 3a) } \\
\text { Daughter cysts may be present within a solid matrix (CE 3b) } \\
\text { Cysts may be less rounded due decrease of intra-cystic fluid }\end{array}$ \\
\hline CE 4* & $\begin{array}{l}\text { Heterogeneous or hyperechoic degenerative contents } \\
\text { No daughter cysts } \\
\text { 'Ball of wool sign' indicates degenerating membranes }\end{array}$ \\
\hline CE 5* & $\begin{array}{l}\text { Cysts with thick calcified, arch-shaped wall } \\
\text { Produce a cone shaped shadow } \\
\text { Partial or complete calcification }\end{array}$ \\
\hline $\begin{array}{l}\text { CE = cystic echinococcosis; CL = cystic lesion; WHO = World Health Organization } \\
\dagger \text { Features are nothognomonic and further diagnostic imaging or tests are required }\end{array}$ \\
\hline
\end{tabular}




\section{Figure Legends}

Figure 1. Ultrasound showing a CE 1 cyst with echogenic hydatid sand. When the sand is moving in the cyst it forms the 'snowflake' sign

Figure 2. Non-contrast CT demonstrating multiple cystic hydatid cysts with dense pericyst. There is no evidence of membranes, septa or calcification, however unlike a simple hepatic cyst, the pericyst is hyperdense (arrow) compared to the adjacent liver.

Figure 3. T2-weighted MRI showing a hyperintense hydatid cyst in the right lobe of liver with hypointense membranes folding within it.

This article is protected by copyright. All rights reserved. 


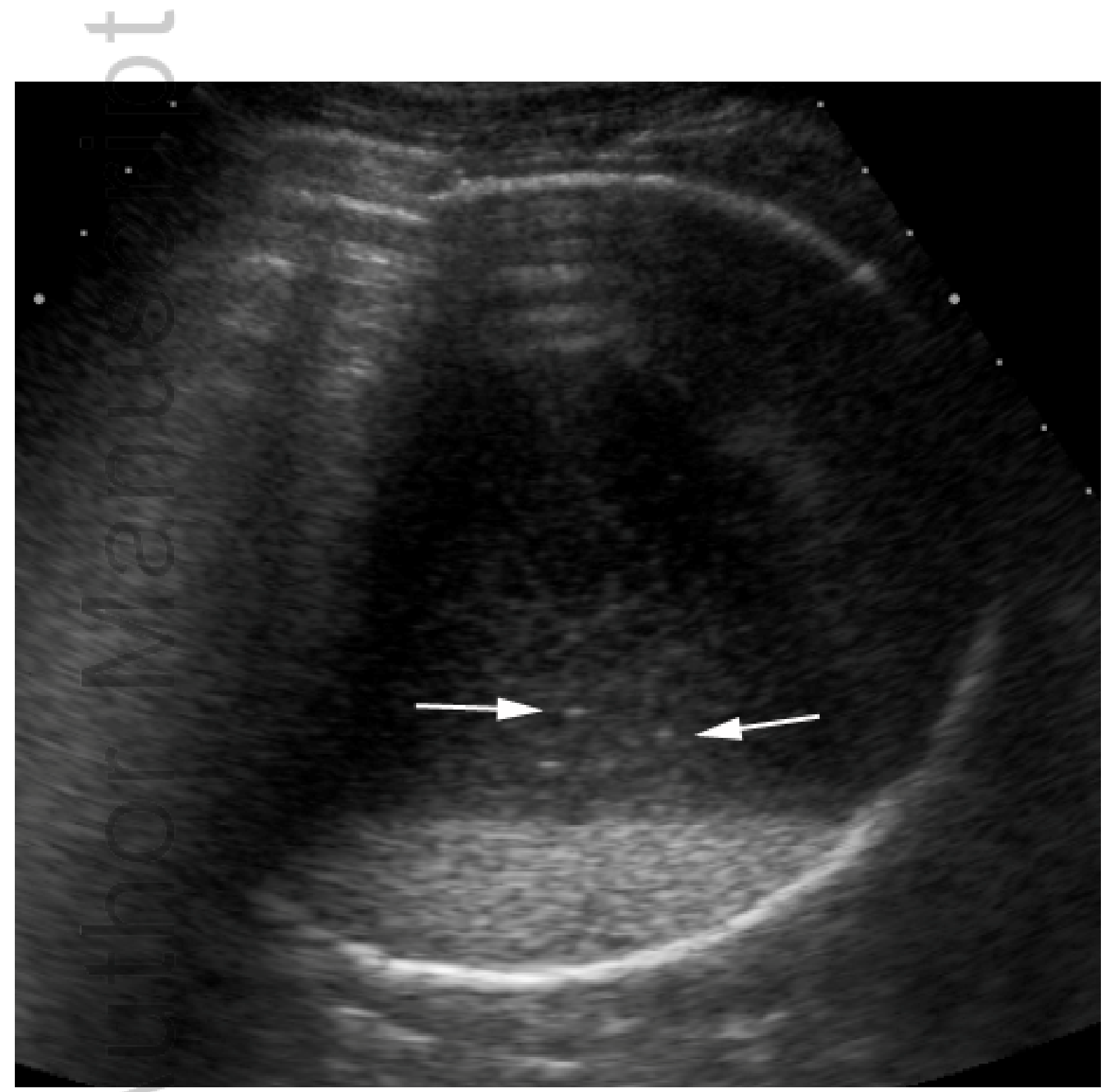

Figure 1.tif

This article is protected by copyright. All rights reserved. 


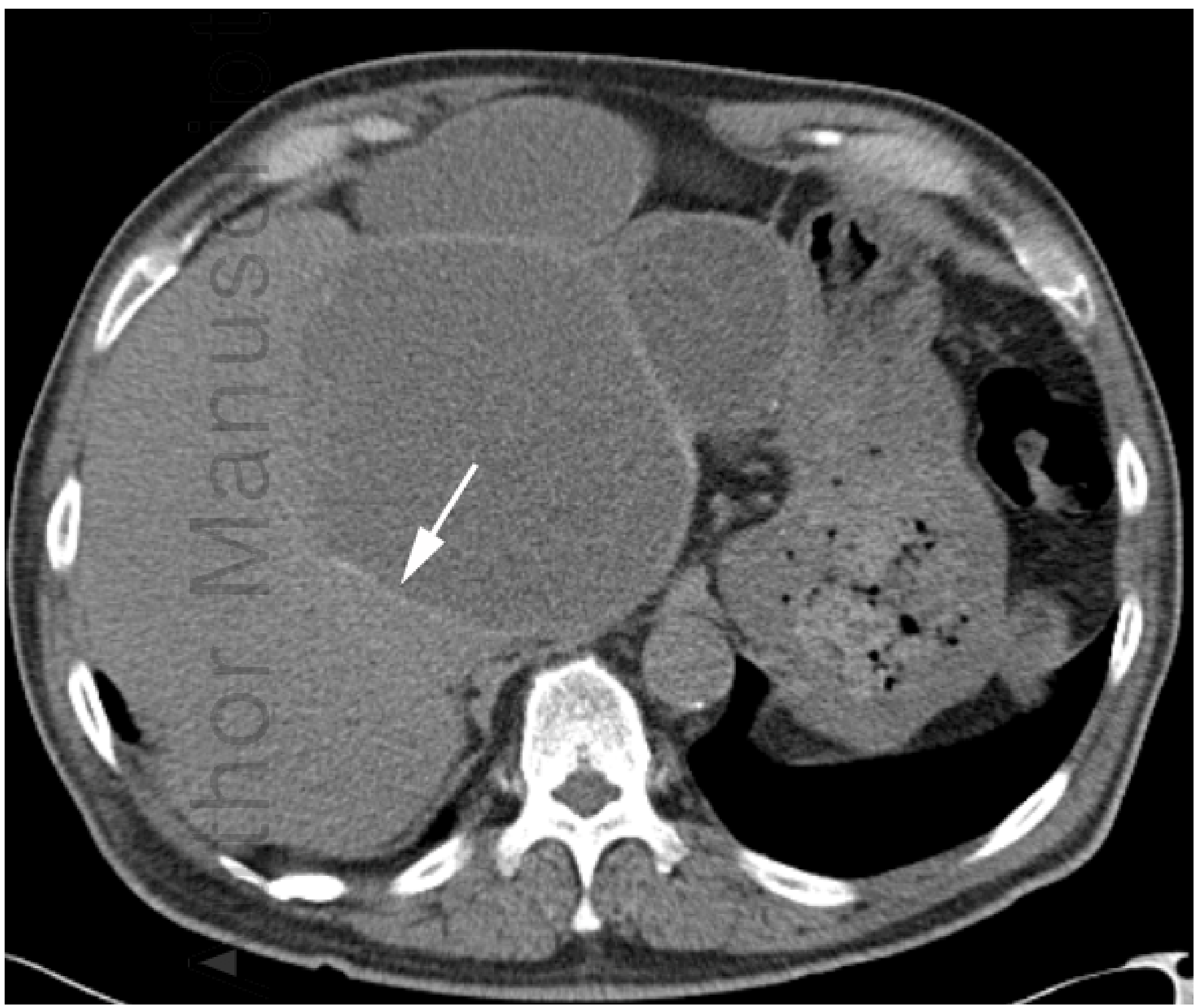

Figure 2.tif

This article is protected by copyright. All rights reserved. 


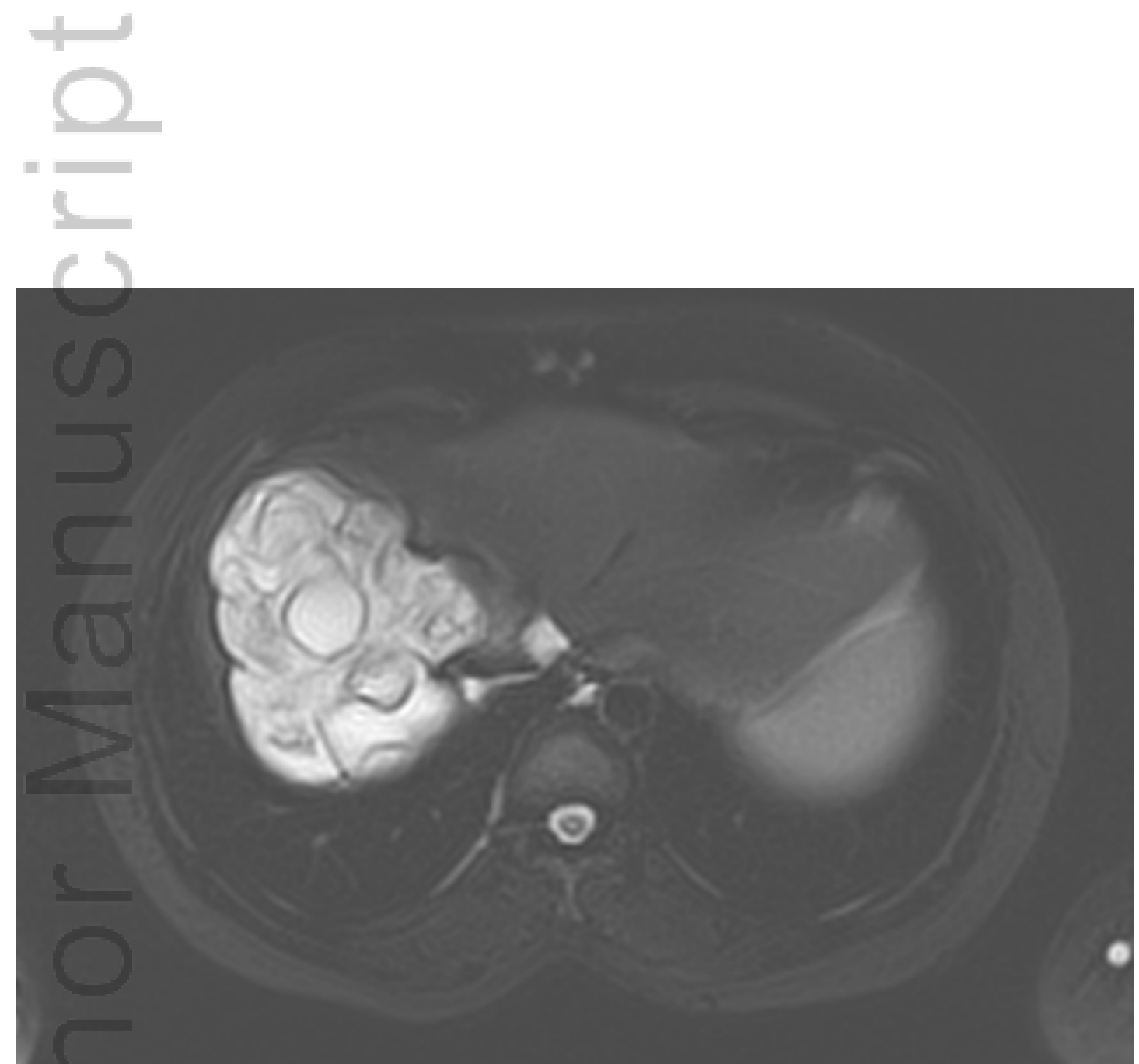

Figure 3.tif

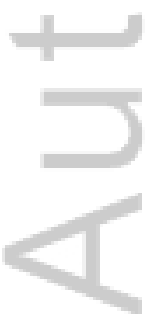

This article is protected by copyright. All rights reserved. 


\section{University Library}

\section{- M M N E R VA A gateway to Melbourne's research publications}

Minerva Access is the Institutional Repository of The University of Melbourne

Author/s:

Keong, B;Wilkie, B;Sutherland, T;Fox, A

Title:

Hepatic cystic echinococcosis in Australia: an update on diagnosis and management

Date:

2018-01-01

Citation:

Keong, B., Wilkie, B., Sutherland, T. \& Fox, A. (2018). Hepatic cystic echinococcosis in Australia: an update on diagnosis and management. ANZ JOURNAL OF SURGERY, 88 (1-2), pp.26-31. https://doi.org/10.1111/ans.14117.

Persistent Link:

http://hdl.handle.net/11343/293566 